\title{
Quadrature Methods for Integral Equations of the Second Kind Over Infinite Intervals
}

\author{
By Ian H. Sloan
}

\begin{abstract}
Convergence results are proved for a class of quadrature methods for integral equations of the form $y(t)=f(t)+\int_{0}^{\infty} k(t, s) y(s) d s$. An important special case is the Nyström method, in which the integral term is approximated by an ordinary quadrature rule. For all of the methods considered here, the rate of convergence is the same, apart from a constant factor, as that of the quadrature approximation to the integral term.
\end{abstract}

1. Introduction. In this paper we analyze a general class of quadrature methods for integral equations of the form

$$
y(t)=f(t)+\int_{0}^{\infty} k(t, s) y(s) d s
$$

under suitable conditions on the kernel $k$ and the inhomogeneous term $f$. It is assumed throughout that the corresponding homogeneous equation has no nontrivial solution.

A similar analysis can also be made for integral equations on the interval $(-\infty, \infty)$. The corresponding results can easily be written down by adapting the results of this paper.

The approximation methods to be considered in this paper are all characterized by an approximate equation of the form

$$
y_{n}(t)=f(t)+\sum_{i=1}^{n} W_{n i}(t) y_{n}\left(s_{n i}\right)
$$

where $s_{n 1}, \ldots, s_{n n}$ are distinct points in $[0, \infty]$, and $W_{n 1}, \ldots, W_{n n}$ are continuous scalar-valued functions on the interval $[0, \infty)$.

The simplest method of this type is the Nyström method [6], in which the integral term of (1.1) is approximated by an ordinary quadrature rule for the interval $[0, \infty)$. In this case, $W_{n i}(t)$ is simply a quadrature weight multiplied by $k\left(t, s_{n i}\right)$; see Section 4.

Remarkably, it appears that no analysis of the Nyström method for an infinite interval has ever been carried out. (See, for example, the comment in [3, pp. 581-582].) Here we first give a general convergence proof and error analysis for approximations of the form (1.2), then specialize to the Nyström method, and finally illustrate with two particular quadrature rules: first, Gauss-Laguerre quadrature, and second, a rule obtained by mapping to a finite interval and then using ordinary Gauss quadrature.

Received April 18, 1980; revised August 11, 1980.

1980 Mathematics Subject Classification. Primary 65R20, 45B05.

Key words and phrases. Integral equation, Fredholm equation, equation of the second kind, infinite interval, Nyström method, quadrature method. 
Any approximation of the form (1.2) is easy to use in practice, once the quantities $W_{n i}(t)$ are known. The first step is to set $t=s_{n j}, j=1, \ldots, n$, to obtain the set of linear equations

$$
\sum_{i=1}^{n}\left[\delta_{j i}-W_{n i}\left(s_{n j}\right)\right] y_{n}\left(s_{n i}\right)=f\left(s_{n j}\right), \quad j=1, \ldots, n,
$$

which may be solved by any convenient method. Then the value of $y_{n}(t)$ at an arbitrary point $t$ may be obtained by the direct use of (1.2).

A previous paper by Atkinson [2] is also concerned with the integral equation (1.1), but is devoted to a different method of approximation, namely that obtained by cutting off the infinite interval at an upper limit $R$ and then letting $R \rightarrow \infty$. In practice, the resulting equation for the finite interval must then be solved approximately for a sequence of values of $R$, usually by conventional quadrature methods. In our opinion, the direct application of quadrature methods over the infinite interval is usually preferable under the conditions assumed in the present paper. On the other hand, Atkinson's analysis covers cases that are outside the scope of the present work: in particular, it covers equations of the Wiener-Hopf type (i.e. $k(t, s)=\kappa(t-s)$ ). The restrictions on $k$ in the present work are stated below.

2. A Theoretical Setting. An awkward problem when considering equations over infinite intervals is the choice of the space of functions within which the solution $y$ is to be sought. In the present work we assume that $y$ and $f$ belong to the space $C_{l}$, the space of continuous functions on $[0, \infty)$ having a limit as $t \rightarrow \infty$. If $g \in C_{l}$, then $g(\infty)$ denotes $\lim g(t)$ as $t \rightarrow \infty$. The space $C_{l}$ is equipped with the norm

$$
\|g\|_{\infty}=\sup _{t \in[0, \infty)}|g(t)| \text {. }
$$

With this norm it is a Banach space, since it is a closed subspace of the Banach space $C$ of all bounded continuous functions on $[0, \infty)$. The notation $C_{l}$ is that of Corduneanu [4].

We shall assume that the kernel $k$ satisfies the conditions

$$
\int_{0}^{\infty}|k(t, s)| d s<\infty
$$

and

$$
\lim _{t^{\prime} \rightarrow t} \int_{0}^{\infty}\left|k\left(t^{\prime}, s\right)-k(t, s)\right| d s=0
$$

for all $t \in[0, \infty)$, and also the condition

$$
\lim _{t \rightarrow \infty} \sup _{t^{\prime}>t} \int_{0}^{\infty}\left|k\left(t^{\prime}, s\right)-k(t, s)\right| d s=0 .
$$

We shall see below that a consequence of these conditions is that

$$
\sup _{t \in[0, \infty)} \int_{0}^{\infty}|k(t, s)| d s<\infty
$$

and from this it follows easily that kernels of the form $k(t, s)=\kappa(t s)$ are excluded. Also, the third condition can easily be seen to exclude kernels of the Wiener-Hopf form $k(t, s)=\kappa(t-s)$. 
The properties assumed for the kernel are motivated by the following result:

Lemмa. The integral operator $K$ defined by

$$
K g(t)=\int_{0}^{\infty} k(t, s) g(s) d s, \quad g \in C_{l},
$$

is a compact operator from $C_{l}$ to itself if and only if (2.1), (2.2) and (2.3) hold.

It follows from the lemma, and from the Fredholm alternative, that (1.1) has a solution $y \in C_{l}$ for each $f \in C_{l}$. It also follows that $I-K$, where $I$ denotes the identity operator, has a bounded inverse on $C_{l}$. (The latter is a stability property, since it ensures that small changes in $f$ produce only small changes in $y$.)

Proof of Lemma. $(\Leftarrow)$ Assume that (2.1), (2.2), and (2.3) hold. We first prove that $K$ maps $C_{l}$ into itself. If $g \in C_{l}$ and $t^{\prime}, t \in[0, \infty)$, we have

$$
\begin{aligned}
\left|K g\left(t^{\prime}\right)-K g(t)\right| & =\left|\int_{0}^{\infty}\left[k\left(t^{\prime}, s\right)-k(t, s)\right] g(s) d s\right| \\
& \leqslant \int_{0}^{\infty}\left|k\left(t^{\prime}, s\right)-k(t, s)\right| d s\|g\|_{\infty} .
\end{aligned}
$$

The last line converges to 0 as $t^{\prime} \rightarrow t$ by virtue of (2.2), hence $K g$ is continuous on $[0, \infty)$. Similarly, by virtue of $(2.3)$, we have

$$
\lim _{t \rightarrow \infty} \sup _{t^{\prime}>t}\left|K g\left(t^{\prime}\right)-K g(t)\right|=0,
$$

from which it follows that $K g(t)$ has a limit as $t \rightarrow \infty$. Thus, $K g \in C_{l}$.

Next, we show that $K$ is a bounded operator on $C_{l}$. It is easily seen that $\|K\|=\|\kappa\|_{\infty}$, where $\kappa(t)=\int_{0}^{\infty}|k(t, s)| d s$. Now it follows, with the aid of elementary inequalities, that

$$
\left|\kappa\left(t^{\prime}\right)-\kappa(t)\right|<\int_{0}^{\infty}\left|k\left(t^{\prime}, s\right)-k(t, s)\right| d s,
$$

hence, from (2.2) and (2.3), we deduce $\kappa \in C_{l}$. Hence, $\|\kappa\|_{\infty}<\infty$, and therefore $\|K\|<\infty$. (Note that the boundedness of $K$ is equivalent to (2.4).)

To show that $K$ is compact, we must show that $K$ maps the unit ball in $C_{l}$ into a relatively compact set, i.e. into a set with compact closure in $C_{l}$. Therefore a necessary first step is to identify the relatively compact subsets of $C_{l}$.

Adapting an argument of Atkinson [2], we may identify the relatively compact subsets of $C_{l}$ by observing that the map $T: C[0,1] \rightarrow C_{l}$, defined by $\operatorname{Th}(t)=$ $h(t /(1+t))$ for $t \in[0, \infty)$, is an isometric isomorphism. Thus, the relatively compact subsets of $C_{l}$ are simply the images under $T$ of the relatively compact subsets of $C[0,1]$. But by the Arzelà-Ascoli theorem, the latter are just the bounded, equicontinuous subsets of $C[0,1]$.

We recall that a subset $S \subset C[0,1]$ is equicontinuous if, given $\varepsilon>0$, there exists $\delta>0$ such that $\left|h\left(x^{\prime}\right)-h(x)\right|<\varepsilon$ for all $x^{\prime}, x \in[0,1]$ satisfying $\left|x^{\prime}-x\right|<\delta$, and all $h \in S$. It is also useful to recall (see, for example, [7, p. 94]) that $S$ is equicontinuous on the compact interval $[0,1]$ if and only if it is equicontinuous at each point of $[0,1]$, i.e. if and only if given $\varepsilon>0$ and $x \in[0,1]$, there exists $\delta(x)$ such that $\left|h\left(x^{\prime}\right)-h(x)\right|<\varepsilon$ for all $x^{\prime} \in[0,1]$ satisfying $\left|x^{\prime}-x\right|<\delta(x)$, and for all $h \in S$. 
We deduce from the preceding two paragraphs that a subset of $C_{l}$ is relatively compact if and only if it is uniformly bounded, equicontinuous at each point $t \in\left[0, \infty\right.$ ), and equiconvergent at $\infty$. (A subset of $C_{l}$ is equiconvergent at $\infty$ if, given $\varepsilon>0$, there exists $T>0$ such that $|g(t)-g(\infty)|<\varepsilon$ for all $t>T$ and all $g$ in the subset.)

Thus, if $B$ denotes the unit ball in $C_{l}$, the operator $K$ is compact if and only if the set $K B$ is equicontinuous at each point of $[0, \infty)$ and equiconvergent at $\infty$. To establish those properties, let $g \in B$ and $t^{\prime}, t \in[0, \infty)$, and consider

$$
\begin{aligned}
\left|K g\left(t^{\prime}\right)-K g(t)\right| & =\left|\int_{0}^{\infty}\left[k\left(t^{\prime}, s\right)-k(t, s)\right] g(s) d s\right| \\
& \leqslant \int_{0}^{\infty}\left|k\left(t^{\prime}, s\right)-k(t, s)\right| d s .
\end{aligned}
$$

Then the equicontinuity of $K B$ at the point $t \in[0, \infty)$ follows from (2.2), and the equiconvergence at $\infty$ follows from (2.3). Thus, the compactness of $K$ is proved.

$(\Rightarrow)$ Now assume that $K$ is a compact operator from $C_{l}$ to itself. Since $K$, in particular, maps the function $g(t) \equiv 1$ into a function in $C_{l}$, the integral $\int_{0}^{\infty} k(t, s) d s$ must be continuous in $t$ and hence exists as a finite number for all $t \in[0, \infty)$. Since a measurable function $h$ is Lebesgue integrable only if $|h|$ is Lebesgue integrable, it follows that $\int_{0}^{\infty}|k(t, s)| d s$ also exists as a finite number for all $t \in[0, \infty)$. Thus, $(2.1)$ is proved.

Also, because $K$ is a compact operator, the set $K B$ is equicontinuous at each point of $[0, \infty)$ and also equiconvergent at $\infty$. From the first it follows that

$$
\lim _{t^{\prime} \rightarrow t} \sup _{g \in B}\left|K g\left(t^{\prime}\right)-K g(t)\right|=0
$$

for all $t \in[0, \infty)$, and from the second that

$$
\lim _{t \rightarrow \infty} \sup _{t^{\prime}>t} \sup _{g \in B}\left|K g\left(t^{\prime}\right)-K g(t)\right|=0 .
$$

But it is easily seen that

$$
\sup _{g \in B}\left|K g\left(t^{\prime}\right)-K g(t)\right|=\int_{0}^{\infty}\left|k\left(t^{\prime}, s\right)-k(t, s)\right| d s,
$$

hence (2.2) and (2.3) follow immediately from (2.5) and (2.6). Q.E.D.

3. Convergence Theory. We shall assume that the quantities $W_{n i}$ in (1.2) satisfy the following three conditions:

$$
\lim _{n \rightarrow \infty} \sum_{i=1}^{n} W_{n i}(t) g\left(s_{n i}\right)=\int_{0}^{\infty} k(t, s) g(s) d s
$$

for all $g \in C_{l}$ and all $t \in[0, \infty)$;

$$
\lim _{t^{\prime} \rightarrow t} \sup _{n} \sum_{i=1}^{n}\left|W_{n i}\left(t^{\prime}\right)-W_{n i}(t)\right|=0
$$

for all $t \in[0, \infty)$; and

$$
\lim _{t \rightarrow \infty} \sup _{t^{\prime}>t} \sup _{n} \sum_{i=1}^{n}\left|W_{n i}\left(t^{\prime}\right)-W_{n i}(t)\right|=0 .
$$


The properties assumed above ensure that the approximate integral operators $K_{n}$, defined for $n \geqslant 1$ by

$$
K_{n} g(t)=\sum_{i=1}^{n} W_{n i}(t) g\left(s_{n i}\right), \quad t \in[0, \infty),
$$

are well behaved in the following sense.

LeMma. The sequence $\left\{K_{n}\right\}$ is a collectively compact set of operators from $C_{l}$ to itself, with the property that $\left\|K_{n} g-K g\right\|_{\infty} \rightarrow 0$ as $n \rightarrow \infty$ for all $g \in C_{l}$, if and only if (3.1), (3.2), and (3.3) hold.

The lemma is proved below. Given the lemma, the following convergence theorem, which is the foundation for all of the results of this paper, is a direct consequence of Anselone's collectively compact operator approximation theory; see [1, Theorem 1.6]. For it follows from the latter that the operators $\left(I-K_{n}\right)^{-1}$ exist and are uniformly bounded for all $n$ sufficiently large, and the error estimate in the theorem then follows from the identity

$$
y_{n}-y=\left(I-K_{n}\right)^{-1}\left(K_{n} y-K y\right) \text {. }
$$

THEOREM 1. Given (2.1)-(2.3) and (3.1)-(3.3), there exists $n_{0} \geqslant 1$ such that $y_{n}$ exists and is unique for all $n>n_{0}$. Moreover, $y_{n}$ converges uniformly to $y$, and there exists $c>0$ such that

$$
\left\|y_{n}-y\right\|_{\infty} \leqslant c\left\|K_{n} y-K y\right\|_{\infty}
$$

for all $n>n_{0}$.

The importance of this theorem lies firstly in the fact that it establishes the existence of the approximate solution $y_{n}$ for all $n$ sufficiently large, and secondly in the guarantee that it provides for the rate of convergence: it assures us that the rate of convergence is the same, apart from a constant factor, as that of the quadrature approximation $\Sigma W_{n i}(t) y\left(s_{n i}\right)$ to the integral term of (1.1).

Proof of Lemma. $(\Leftarrow)$ Assume that (3.1), (3.2) and (3.3) hold. We first show that $K_{n}$ maps $C_{l}$ into itself. If $g \in C_{l}$ and $t^{\prime}, t \in[0, \infty)$, then

$$
\begin{aligned}
\left|K_{n} g\left(t^{\prime}\right)-K_{n} g(t)\right| & =\left|\sum_{i=1}^{n}\left[W_{n i}\left(t^{\prime}\right)-W_{n i}(t)\right] g\left(s_{n i}\right)\right| \\
& <\sum_{i=1}^{n}\left|W_{n i}\left(t^{\prime}\right)-W_{n i}(t)\right|\|g\|_{\infty},
\end{aligned}
$$

the right-hand side of which converges to zero as $t^{\prime} \rightarrow t$ for all $t \in[0, \infty)$ by virtue of (3.2). Hence, $K_{n} g$ is continuous on [0, ). Similarly, $K_{n} g(t)$ has a limit as $t \rightarrow \infty$ by virtue of (3.3). Hence, $K_{n} g \in C_{l}$.

Next, we show that $K_{n}$ is a bounded operator on $C_{l}$. It is easily seen that

$$
\left\|K_{n}\right\|=\sup _{t \in[0, \infty)} \kappa_{n}(t)=\left\|\kappa_{n}\right\|_{\infty}
$$

where

$$
\kappa_{n}(t)=\sum_{i=1}^{n}\left|W_{n i}(t)\right|
$$


Now, from (3.2) and (3.3) it follows, by an argument similar to that in the preceding paragraph, that $\kappa_{n} \in C_{l}$. Hence, $\left\|K_{n}\right\|=\left\|\kappa_{n}\right\|_{\infty}<\infty$.

We recall that the set $\left\{K_{n}\right\}$ of bounded linear operators on the Banach space $C_{l}$ is collectively compact in the sense of [1] if the set $S$, defined by

$$
S=\left\{K_{n} g: g \in B, n>1\right\},
$$

is a relatively compact subset of $C_{l}$. (Here $B$ is the unit ball in $C_{l}$.) From the discussion in the preceding section, we may deduce that the set $\left\{K_{n}\right\}$ is collectively compact if $S$ is bounded, equicontinuous at each point $t \in[0, \infty)$, and equiconvergent at $\infty$.

To prove the equicontinuity and equiconvergence properties of $S$, let $g \in B$ and $t^{\prime}, t \in[0, \infty)$, and consider, for $m \geqslant 1$,

$$
\begin{aligned}
\left|K_{m} g\left(t^{\prime}\right)-K_{m} g(t)\right| & =\left|\sum_{i=1}^{m}\left[W_{m i}\left(t^{\prime}\right)-W_{m i}(t)\right] g\left(s_{m i}\right)\right| \\
& \leqslant \sum_{i=1}^{m}\left|W_{m i}\left(t^{\prime}\right)-W_{m i}(t)\right|<\sup _{n} \sum_{i=1}^{n}\left|W_{n i}\left(t^{\prime}\right)-W_{n i}(t)\right| .
\end{aligned}
$$

The right-hand side is independent of $m$ and of $g$, and converges to zero as $t^{\prime} \rightarrow t$ by virtue of (3.2). Hence, $S$ is equicontinuous at each point $t \in[0, \infty)$. Similarly, by virtue of (3.3), $S$ is equiconvergent at $\infty$.

Next, we seek to show that for all $g \in C_{l}$ we have $\left\|K_{n} g-K g\right\|_{\infty} \rightarrow 0$ as $n \rightarrow \infty$. Now, (3.1) tells us that $K_{n} g(t) \rightarrow K g(t)$ for all $t \in[0, \infty)$, and hence it only remains to prove that this convergence is uniform. It is known (see, for example, $[1$, p. 7]) that pointwise convergence of an equicontinuous family on a compact interval is sufficient to ensure uniform convergence. It follows that pointwise convergence on the interval $[0, \infty)$ of a family that is equicontinuous at each point of $[0, \infty)$ and equiconvergent at $\infty$ is sufficient to ensure uniform convergence. (This may be deduced by using the previously noted isomorphism between $C_{l}$ and $C[0,1]$.) But the equicontinuity and equiconvergence properties have already been established for the sequence $\left\{K_{n} g\right\}$. Hence, we conclude that

$$
\lim _{n \rightarrow \infty}\left\|K_{n} g-K g\right\|_{\infty}=0
$$

for all $g \in C_{l}$.

Finally, we show that the set $S$ is bounded. From (3.5) we have

$$
\sup _{n}\left\|K_{n} g\right\|_{\infty}<\infty
$$

for all $g \in C_{l}$, hence, since $\left\{K_{n}\right\}$ is a sequence of bounded operators on the Banach space $C_{l}$, it follows from the uniform-boundedness (Banach-Steinhaus) theorem that

$$
\sup _{n}\left\|K_{n}\right\|<\infty
$$

The left-hand side is a bound for the set $S$. Thus, $S$ is bounded, equicontinuous at each point of $[0, \infty)$, and equiconvergent at $\infty$, and therefore relatively compact. Hence, the set $\left\{K_{n}\right\}$ is collectively compact.

$(\Rightarrow)$ Assume that $\left\{K_{n}\right\}$ is collectively compact, and that $\left\|K_{n} g-K g\right\|_{\infty} \rightarrow 0$ as $n \rightarrow \infty$ for all $g \in C_{l}$. The second property implies (3.1). The first implies that the 
set $S$, defined by (3.4), is equicontinuous at each point of $[0, \infty)$, i.e.

$$
\lim _{t^{\prime} \rightarrow t} \sup _{n} \sup _{g \in B}\left|K_{n} g\left(t^{\prime}\right)-K_{n} g(t)\right|=0
$$

for all $t \in[0, \infty)$, and also that $S$ is equiconvergent at $\infty$, from which it follows that

$$
\lim _{t \rightarrow \infty} \sup _{t^{\prime}>t} \sup _{n} \sup _{g \in B}\left|K_{n} g\left(t^{\prime}\right)-K_{n} g(t)\right|=0 .
$$

But it is easily seen that

$$
\sup _{g \in B}\left|K_{n} g\left(t^{\prime}\right)-K_{n} g(t)\right|=\sum_{i=1}^{n}\left|W_{n i}\left(t^{\prime}\right)-W_{n i}(t)\right| .
$$

Hence, (3.2) and (3.3) follow immediately from (3.6) and (3.7). Q.E.D.

4. The Nyström Method. In the Nyström method [6], one approximates the integral term of (1.1) by a quadrature rule of the form

$$
\int_{0}^{\infty} z(s) d s \approx \sum_{i=1}^{n} \omega_{n i} z\left(s_{n i}\right)
$$

Specific examples of such quadrature rules are discussed in the following two sections.

With the integral term of (1.1) approximated by (4.1), it is clear that one does indeed obtain an approximation of the form (1.2), with $W_{n i}$ now given by

$$
W_{n i}(t)=\omega_{n i} k\left(t, s_{n i}\right) \text {. }
$$

The Nyström method can be brought within the scope of Theorem 1 if the rule (4.1) has the property that

$$
\lim _{n \rightarrow \infty} \sum_{i=1}^{n} \omega_{n i} k\left(t, s_{n i}\right) g\left(s_{n i}\right)=\int_{0}^{\infty} k(t, s) g(s) d s
$$

for all $t \in[0, \infty)$ and all $g \in C_{l}$. In words, the property is that the rule (4.1) should converge to the exact result when the rule is applied to the integral term of (1.1) with $y$ replaced by any function $g \in C_{l}$. If that property holds, and if $k$ satisfies certain conditions, then Theorem 1 is applicable.

To allow us to specify the conditions on $k$, it is convenient to introduce a function $H \in L_{1}(0, \infty)$ having the property that

$$
\lim _{n \rightarrow \infty} \sum_{i=1}^{n} \omega_{n i} H\left(s_{n i}\right) g\left(s_{n i}\right)=\int_{0}^{\infty} H(s) g(s) d s
$$

for all $g \in C_{l}$. (A function $H$ with this property certainly exists, since we may take $H(s)=k\left(t_{0}, s\right)$ for fixed $t_{0} \in[0, \infty)$. In practice one should choose an $H$ that approaches zero as slowly as possible as $s \rightarrow \infty$.)

Let us now define

$$
r_{t}(s)=r(t, s)=k(t, s) / H(s) .
$$

We shall show that for Theorem 1 to be applicable it is sufficient that $r_{t}$ satisfies each of

$$
r_{t} \in C_{l}
$$


and

$$
\lim _{t^{\prime} \rightarrow t}\left\|r_{t^{\prime}}-r_{t}\right\|_{\infty}=0
$$

for all $t \in[0, \infty)$, and also

$$
\lim _{t \rightarrow \infty} \sup _{t^{\prime}>t}\left\|r_{t^{\prime}}-r_{t}\right\|_{\infty}=0
$$

To show the sufficiency of (4.5) to (4.7), first note that, because $k(t, s)=$ $H(s) r_{t}(s)$, the properties (2.1), (2.2), and (2.3) that must be satisfied by the kernel follow immediately from (4.5), (4.6), and (4.7), respectively.

Next, it follows from (4.2) and (4.4), for $g \in C_{l}$ and $t \in[0, \infty)$, that

$$
\begin{aligned}
\lim _{n \rightarrow \infty} \sum_{i=1}^{n} W_{n i}(t) g\left(s_{n i}\right) & =\lim _{n \rightarrow \infty} \sum_{i=1}^{n} \omega_{n i} k\left(t, s_{n i}\right) g\left(s_{n i}\right) \\
& =\lim _{n \rightarrow \infty} \sum_{i=1}^{n} \omega_{n i} H\left(s_{n i}\right) r_{t}\left(s_{n i}\right) g\left(s_{n i}\right) \\
& =\int_{0}^{\infty} H(s) r_{t}(s) g(s) d s=\int_{0}^{\infty} k(t, s) g(s) d s,
\end{aligned}
$$

where we have used the property (4.3) to evaluate the limit (that property being applicable because $r_{t} g \in C_{l}$ ). Hence, (3.1) is satisfied.

Finally, if $t^{\prime}, t \in[0, \infty)$, we have

$$
W_{n i}\left(t^{\prime}\right)-W_{n i}(t)=\omega_{n i} H\left(s_{n i}\right)\left[r_{t^{\prime}}\left(s_{n i}\right)-r_{t}\left(s_{n i}\right)\right],
$$

so that

$$
\begin{aligned}
\sum_{i=1}^{n}\left|W_{n i}\left(t^{\prime}\right)-W_{n i}(t)\right| & \leqslant \sum_{i=1}^{n}\left|\omega_{n i}\right|\left\|H\left(s_{n i}\right)\right\| r_{t^{\prime}}-r_{t} \|_{\infty} \\
& \leqslant M\left\|r_{t^{\prime}}-r_{t}\right\|_{\infty}
\end{aligned}
$$

where

$$
M=\sup _{n} \sum_{i=1}^{n}\left|\omega_{n i}\right|\left|H\left(s_{n i}\right)\right| .
$$

That $M$ is finite follows from observing that the left-hand side of (4.3) is the limit of a sequence of bounded linear functionals on the space $C_{l}$ and then invoking the uniform-boundedness theorem. Then, by using (4.8), we may deduce the desired properties (3.2) and (3.3) from the respective assumptions (4.6) and (4.7).

Since all the conditions of Theorem 1 have now been established, the following result is a corollary of Theorem 1 .

Corollary 1. Assume that $H \in L_{1}(0, \infty)$, and that the limit (4.3) holds for all $g \in C_{l}$. Moreover, assume that the function $r_{t}$, defined by $r_{t}(s)=k(t, s) / H(s)$, satisfies (4.5), (4.6), and (4.7). Then the conclusions of Theorem 1 hold for the Nyström method based on the quadrature rule (4.1).

5. Gauss-Laguerre Quadrature. To illustrate the Nyström method, we first consider the case of Gauss-Laguerre quadrature; see, for example, [5, p. 173]. The rule may be stated as

$$
\int_{0}^{\infty} e^{-s} u(s) d s \approx \sum_{i=1}^{n} \mu_{n i} u\left(s_{n i}\right)
$$


where $s_{n 1}, \ldots, s_{n n}$ are the zeros of the $n$th degree Laguerre polynomial $L_{n}(s)$, and the weights $\mu_{n i}$ are such that the rule is exact if $u$ is any polynomial of degree $<2 n$.

The rule can easily be expressed in the form (4.1), by setting $z(s)=e^{-s} u(s)$. The weights $\omega_{n i}$ in the rule (4.1) are then given by $\omega_{n i}=\mu_{n i} \exp \left(s_{n i}\right)$.

A sufficient condition for convergence of the Gauss-Laguerre rule to the exact integral (see [12, p. 559]) is that $u$ in $(5.1)$ be continuous on $[0, \infty)$, and satisfy

$$
|u(s)| \leqslant M e^{s} / s^{1+e}, \quad s \in[0, \infty)
$$

for some positive numbers $M$ and $\varepsilon$.

It follows that for the case of Gauss-Laguerre quadrature we may choose the function $H$ of the preceding section to be

$$
H(s)=(1+s)^{-1-e},
$$

where $\varepsilon$ is an arbitrary positive number-for the convergence result in the preceding paragraph then assures us that the limit property (4.3) holds for all $g \in C_{l}$. We deduce the following as a special case of Corollary 1.

Corollary 2. Assume that for some positive number $\varepsilon$ the function $r_{t}$, defined by $r_{t}(s)=(1+s)^{1+\varepsilon} k(t, s)$, satisfies (4.5), (4.6), and (4.7). Then the conclusions of Theorem 1 hold for the Nyström method based on Gauss-Laguerre quadrature.

As a numerical example, we consider the integral equation

$$
y(t)=f(t)+\int_{0}^{\infty} \frac{1}{s^{2}+t^{2}+1} y(s) d s,
$$

with

$$
f(t)=\frac{1}{t^{2}+1}-\frac{\pi}{2 t^{2}}\left[1-\left(t^{2}+1\right)^{-1 / 2}\right],
$$

the exact solution of which is

$$
y(t)=\frac{1}{t^{2}+1} .
$$

It is clear that $f \in C_{l}$. Moreover, it can be shown that the function

$$
r_{t}(s)=(1+s)^{3 / 2}\left(s^{2}+t^{2}+1\right)^{-1}
$$

satisfies (4.5) to (4.7), thus the conditions of Corollary 2 are satisfied with $\varepsilon=1 / 2$.

Numerical results for this example are shown in Table 1. Evidently, the rate of convergence is rather slow. The reason is not hard to find: the convergence result above only asserts that the rate of convergence of $y_{n}$ to $y$ is the same, apart from a constant factor, as that of the Gauss-Laguerre quadrature rule applied to the integral term of (1.1); but the Gauss-Laguerre rule is not at all appropriate for this particular integral, because the integral does not have an exponentially decaying character. In fact, for such an integral, with an integrand of rational character, it is probably better to proceed as in the following section, by transforming the integral to a finite interval and then using an ordinary quadrature rule. 


\section{TABLE 1}

Error norms for the Nyström method with Gauss-Laguerre quadrature

\begin{tabular}{cl}
\hline$n$ & Error norm \\
\hline 4 & 0.20 \\
9 & $0.18(-1)$ \\
12 & $0.53(-3)$ \\
15 & $0.19(-2)$ \\
18 & $0.61(-3)$ \\
21 & $0.33(-4)$ \\
\hline
\end{tabular}

6. Mapping to a Finite Interval. Again the Nyström method is used, but this time the quadrature rule (4.1) is obtained by transforming the integral to a finite interval and then using an ordinary quadrature rule. To be definite, let us use the change of variable formula

$$
s=\frac{x}{1-x}, \quad 0<x<1
$$

so that the integral becomes

$$
\int_{0}^{\infty} z(s) d s=\int_{0}^{1} z\left(\frac{x}{1-x}\right) \frac{d x}{(1-x)^{2}},
$$

and for the quadrature rule over the interval $[0,1]$ let us use ordinary Gauss quadrature.

Then the right-hand side of (6.2) is approximated by the Gauss rule

$$
\int_{0}^{1} m(x) d x \approx \sum_{i=1}^{n} \mu_{n i} m\left(x_{n i}\right)
$$

where $x_{n 1}, \ldots, x_{n n}$ are the zeros of the $n$th degree Legendre polynomial shifted to $[0,1]$, and the weights $\mu_{n i}$ are such that the rule is exact if $m$ is any polynomial of degree $<2 n$. On combining (6.2) and (6.3), the quadrature rule for the infinite integral becomes

$$
\int_{0}^{\infty} z(s) d s \approx \sum_{i=1}^{n} \omega_{n i} z\left(s_{n i}\right)
$$

where

$$
s_{n i}=\frac{x_{n i}}{1-x_{n i}} \quad \text { and } \quad \omega_{n i}=\frac{\mu_{n i}}{\left(1-x_{n i}\right)^{2}}
$$

To apply the convergence result of Section 4, one needs to specify the function $H$. The Gauss quadrature rule (6.3) is known to converge to the exact result as $n \rightarrow \infty$ for all $m \in C[0,1]$ (see [5, p. 99]), hence a possible choice for $H$ is

$$
H(s)=(1+s)^{-2},
$$

for it can easily be seen that the property (4.3) then holds for all $g \in C_{l}$. However, if we make that choice for $H$ in Corollary 1 , then it turns out that the corresponding conditions on the kernel are somewhat restrictive and are in fact not satisfied by a particular integral equation that we wish to consider (namely (5.2)). 
The condition on the kernel can be weakened if we take advantage of the fact (see [8]) that Gauss quadrature converges even for certain functions with endpoint singularities. Precisely, the following result, which is a slight extension of a result in [8], proves to be useful for our purposes:

LEMMA. The Gauss quadrature rule (6.3) converges to the exact result as $n \rightarrow \infty$ if $m$ is of the form

$$
m(x)=p(x)(1-x)^{-\tau},
$$

where $p$ is bounded and continuous on the interval $[0,1)$, and $\tau<1$.

Proof. Let $m$ be given by (6.4), and let $I_{n}(m)$ denote the Gauss quadrature rule in (6.3) and $I(m)$ the exact integral. To prove that $I_{n}(m) \rightarrow I(m)$ as $n \rightarrow \infty$, let $\varepsilon>0$ be given, and then choose $\delta>0$ so that

$$
\int_{1-\delta}^{1}(1-x)^{-\tau} d x<\varepsilon / 6\|p\|_{\infty}
$$

where the integral on the left is an improper integral if $0<\tau<1$. Now let us decompose $m$ into two pieces $m_{1}$ and $m_{2}$, defined by

$$
m_{1}(x)= \begin{cases}m(x), & x<1-\delta \\ 0, & x>1-\delta\end{cases}
$$

and

$$
m_{2}(x)=m(x)-m_{1}(x)
$$

Then $m_{1}$ is piecewise continuous on $[0,1]$, so it is certainly true (see $[5$, p. 102]) that $I_{n}\left(m_{1}\right) \rightarrow I\left(m_{1}\right)$. Thus, for all $n$ sufficiently large, we have

$$
\left|I_{n}\left(m_{1}\right)-I\left(m_{1}\right)\right|<\varepsilon / 2
$$

On the other hand,

$$
\begin{aligned}
\left|I_{n}\left(m_{2}\right)-I\left(m_{2}\right)\right| & \leqslant\left|I_{n}\left(m_{2}\right)\right|+\left|I\left(m_{2}\right)\right| \\
& =\left|\sum_{i=1}^{n} \mu_{n i} m_{2}\left(x_{n i}\right)\right|+\left|\int_{0}^{1} m_{2}(x) d x\right| \\
& \leqslant\|p\|_{\infty}\left[\sum_{x_{n i}>1-\delta} \frac{\mu_{n i}}{\left(1-x_{n i}\right)^{\tau}}+\int_{1-\delta}^{1} \frac{1}{(1-x)^{\tau}} d x\right] .
\end{aligned}
$$

Now it follows from a theorem of Rabinowitz [8] that the Gauss rule converges to the exact result for the particular function $(1-x)^{-\tau}$, hence it also converges to the exact result for the same function multiplied by a step function. Thus, for all $n$ sufficiently large, we have

$$
\begin{aligned}
\left|I_{n}\left(m_{2}\right)-I\left(m_{2}\right)\right| & \leqslant\|p\|_{\infty}\left[2 \int_{1-\delta}^{1} \frac{1}{(1-x)^{\tau}} d x+\int_{1-\delta}^{1} \frac{1}{(1-x)^{\tau}} d x\right] \\
& <\varepsilon / 2 .
\end{aligned}
$$

Since $m=m_{1}+m_{2}$, for all $n$ sufficiently large we have

$$
\left|I_{n}(m)-I(m)\right|<\varepsilon,
$$

and the proof of the lemma is complete. Q.E.D. 
We may now choose the function $H$ of Section 4 to be

$$
H(s)=(1+s)^{-1-e},
$$

where $\varepsilon$ is any positive number, since the necessary convergence property (4.3) then follows from the lemma. The following result, which closely parallels Corollary 2 , may then be obtained as a special case of Corollary 1 .

Corollary 3. Assume that for some positive number $\varepsilon$ the function $r_{t}(s)=$ $(1+s)^{1+\varepsilon} k(t, s)$ satisfies (4.5), (4.6), and (4.7). Then the conclusions of Theorem 1 hold for the Nyström method, if the quadrature rule is obtained by the change of variable (6.1), followed by Gauss quadrature.

For a numerical example, we again consider the integral equation defined by (5.2) and (5.3). The conditions of the corollary, as noted in the preceding section, are then satisfied with $\varepsilon=1 / 2$.

The numerical results, given in Table 2, display much faster convergence than the Gauss-Laguerre results of Table 1. The reason is clear: the present quadrature rule is far more appropriate than the Gauss-Laguerre rule for the integral term of this particular integral equation.

TABLE 2

Error norms for the Nyström method based on mapping to

finite interval followed by Gauss quadrature

\begin{tabular}{cc}
\hline$n$ & Error norm \\
\hline 4 & $0.16(-1)$ \\
8 & $0.19(-4)$ \\
12 & $0.34(-6)$ \\
\hline
\end{tabular}

7. Conclusion. In this paper a convergence proof and error analysis has been given for the Nyström method for an integral equation of the second kind over an infinite interval. Two particular examples of the Nyström method have been discussed in detail, namely that based on Gauss-Laguerre quadrature, and that based on mapping the infinite interval to a finite interval and then using Gauss quadrature.

The main convergence result, Theorem 1, also covers a much more general class of approximation methods, including those based on the use of product-integration rules for the integral term of (1.1). (For a general discussion of product-integration rules see [9].) However, we have not discussed any applications of this kind in the present work, for the very good reason that there are apparently not yet any useful convergence results for product-integration rules over infinite intervals (except for those obtained, as in [9], by transforming finite-interval rules). Nevertheless, such results are expected to be available in the future, perhaps in the style of the finite-interval results of [11], and Theorem 1 is then expected to be applicable. It is likely that the direct application of Theorem 1 to such cases will prove cumbersome, but that a further development of the theory, in parallel to one given for the finite-interval case in [10], will make the application to product-integration rules over infinite intervals quite straightforward. 
8. Acknowledgement. I am indebted to the Institute for Physical Science and Technology and the Department of Physics and Astronomy at the University of Maryland for their hospitality at the time this work was carried out. I am also indebted to Professor P. M. Anselone for his interest and encouragement.

Department of Applied Mathematics

University of New South Wales

Sydney, N.S.W. 2033, Australia

1. P. M. Anselone, Collectively Compact Operator Approximation Theory and Applications to Integral Equations, Prentice-Hall, Englewood Cliffs, N.J., 1971.

2. K. E. AtKInson, "The numerical solution of integral equations on the half-line," SIAM J. Numer. Anal., v. 6, 1969, pp. 375-397.

3. C. T. H. BAKeR, The Numerical Treatment of Integral Equations, Clarendon Press, Oxford, 1977.

4. C. Corduneanu, Integral Equations and Stability of Feedback Systems, Academic Press, New York, 1973.

5. P. J. Davis \& P. Rabinowitz, Methods of Numerical Integration, Academic Press, New York, 1975.

6. E. J. NYSTRÖM, "Über die praktische Auflösung von Integralgleichungen mit Anwendungen auf Randwertaufgaben,” Acta Math., v. 54, 1930, pp. 185-204.

7. J. D. PRYCE, Basic Methods of Linear Functional Analysis, Hutchinson University Library, London, 1973.

8. P. RABINOWITZ, "Gaussian integration in the presence of a singularity," SIAM J. Numer. Anal., v. 4, 1967, pp. 191-201.

9. I. H. SlonN, "On choosing the points in product integration," J. Math. Phys., v. 21, 1980, pp. $1032-1039$.

10. I. H. SloAN, "Analysis of general quadrature methods for integral equations of the second kind." (Submitted for publication.)

11. W. E. Smith \& I. H. SloAN, "Product-integration rules based on the zeros of Jacobi polynomials," SIAM J. Numer. Anal., v. 17, 1980, pp. 1-13.

12. J. V. USPENSKY, "On the convergence of quadrature formulas related to an infinite interval,"

Trans. Amer. Math. Soc., v. 30, 1928, pp. 542-559. 